\title{
Introduction to Craniosynostosis
}

\author{
John A. Persing, M.D.
}

Skull-shape irregularities have been a subject of interest for centuries. Ancient civilizations intentionally deformed skulls so as to recognize members of their own tribe or identify status within the group. Pathological irregularities in skull shape, related to craniosynostosis, were studied and later grouped with other congenital- and metabolic-related deformities of the skull. Virchow initially ascribed cranial deformities associated with craniosynostosis to cretinism and later attributed premature fusion of the skull suture to inflammation of the meninges. The suspected causes were malaria and syphillis. As understanding of the pathological development of skull deformity increased, the ways in which skull deformities were treated, improved. Metabolic-related deformities are rarely treated by surgical means as the first line of management. However, patients in whom craniosynostosis has caused deformities of the skull and face have been treated by various surgical techniques: initially by the surgical release of the fused sutures (linear craniectomy). These approaches were followed by regional cranioplasties, more extensive and comprehensive bone remodelling, ultimately culminating in techniques such as the whole-vault cranioplasty. In time, questions were raised as to whether deformities of the skull associated with some forms of craniosynostosis might be treatable by less extensive operative procedures. The possibility of the latter type of procedure was in part fueled by technological advances, such as the endoscope and distraction osteogenesis devices, and in part by managed care initiatives emphasizing shorter hospital stays and less extensive surgical care. Procedures such as strip craniectomy, the use of custom-molded skull helmets, and distraction osteogenesis, have evolved as possible solutions to some of these questions; they will be addressed in this issue of Neurosurgical Focus.

Correct treatment requires a correct diagnosis. In this issue Jane, et al., show that appreciation of subtleties of the disease, within one form of craniosynostosis, is necessary to tailor the proper operative procedure to the unique deformity. Different challenges are posed by other forms of skull deformity. Recently, a marked increase in the number of oblique skull deformities has been observed by neurosurgeons and craniofacial surgeons. This increase in cases has been largely brought about the "back to sleep" campaign advocated by the American Academy of Pediatrics, in which it is recommended that children be positioned supine while asleep, to reduce the likelihood of sudden infant death syndrome (SIDS). Although this approach to avoiding fatality has certainly been beneficial and has yielded dramatically reduced rates of SIDS, one unexpected outcome has been a marked increase in the number of skull-shape irregularities. Initially, diagnosis of these skull deformities was confused with that of unilateral lambdoid synostosis; however, subsequent detailed descriptions of the true anatomical characteristics associated with unilateral lambdoid synostosis have since been published. Thus, one may now effectively differentiate craniosynostosis from induced molding skull deformities (for example, deformational plagiocephaly), thus avoiding unnecessary surgery. The number of children in whom an irregular skull shape is related to deformational plagiocephaly, however, continues to grow. Effective strategies are needed to treat these deformities. The American Academy of Pediatrics has recently recommeded that parents continue to allow the newborn child to lie supine, but that they be instructed to turn the newborn's head on alternate sides (right and left) nightly so as to prevent the deformity. Even though this may be effective in reducing future cases of deformational plagiocephaly, currently there are many patients with deformed skulls for whom some form of management is required. Physical therapy is regularly used to correct concomitant torticollis. Skull molding helmets of various designs will be discussed later in this issue of Neurosurgical Focus. Although conceptually, a skull molding cap may be effective in changing the overall shape of the skull, it remains unclear whether deformational plagiocephaly (as that seen in cases in which infants were placed supine to avoid SIDS) is more effectively treated with caps/helmets or physical therapy. There may be a continuum in which the more severe skull deformities are effectively treated with a skull molding cap, and in more mild forms the benefit may be less evident. This issue of Neurosurgical Focus will also address this and how a child who presents at a later age, when the skull is less malleable, may be managed nonoperatively.

Even as the cellular, genetic, and biochemical aspects of craniosynostosis are becoming better known, particularly as they relate to bone development in the skull, we are only now beginning to comprehend the breadth of the abnormality. It is hoped that with this greater understanding, more effective and less dramatic interventions will be made available to our patients. 